\title{
No paradigm changes with checkpoint inhibitor monotherapy in patients with metastatic renal cell carcinoma and brain metastases
}

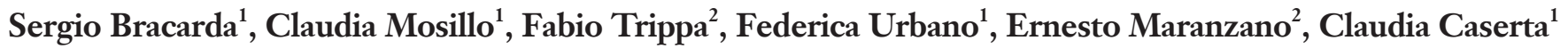 \\ ${ }^{1}$ Medical Oncology Unit, ${ }^{2}$ Radiotherapy Oncology Centre, Azienda Ospedaliera S. Maria, Terni, Italy \\ Correspondence to: Sergio Bracarda, MD. Medical Oncology, Azienda Ospedaliera Santa Maria-Terni, Viale Tristano Di Joannuccio 1, 05100 Terni, \\ Italy. Email: sergiobracarda@gmail.com. \\ Provenance: This is an invited article commissioned by the Section Editor Dr. Xiao Li (Department of Urology, Jiangsu Cancer Hospital, Jiangsu \\ Institute of Cancer Research, Nanjing Medical University Affiliated Cancer Hospital, Nanjing, China). \\ Comment on: Flippot R, Dalban C, Laguerre B, et al. Safety and Efficacy of Nivolumab in Brain Metastases From Renal Cell Carcinoma: Results of \\ the GETUG-AFU 26 NIVOREN Multicenter Phase II Study. J Clin Oncol 2019;37:2008-16.
}

Submitted Oct 25, 2019. Accepted for publication Nov 07, 2019.

doi: $10.21037 / \mathrm{atm} .2019 .11 .50$

View this article at: http://dx.doi.org/10.21037/atm.2019.11.50

The prognosis of patients with metastatic renal cell carcinoma (mRCC) is evaluated by using the International Metastatic Renal Database Consortium risk score. Although the metastatic site is not counted in the prognostic classification, brain, liver, and bone metastases are normally considered as negative prognosticators (1).

About $10 \%$ of patients with mRCC will develop brain metastases $(\mathrm{BM})$ along their natural history, with a life expectancy if not treated, of about 4-6 months (2). Unfortunately, validated therapeutic approaches for these patients are still lacking, because mRCC cases with untreated BM are normally excluded from regulatory phase III trials.

The largest experience in this setting derives from the sunitinib expanded access program (EAP) (3). Out of the overall sunitinib EAP study population, BM were observed in $7 \%$ of the enrolled patients $(213 / 3,464)$. In these cases, an overall response rate (ORR) of $12 \%$ and a median overall survival (mOS) of 9.2 months was achieved. These results confirmed a worse prognosis of patients with BM compared to patients without BM (mOS 9.2 vs. 18.4 months), but also supported the activity of sunitinib in this subgroup, due to an increased disease control. However, no details were available regarding eventual relationships of prior treatment for BM and local response (4).

In a phase II trial for mRCC patients with encephalic lesions, Chevreau et al. reported few BM responses in patients treated with sunitinib alone (5). Therefore, in presence of $\mathrm{BM}$, a local treatment [surgical metastases resection, whole-brain radiotherapy (WBRT), and stereotactic radiotherapy] should be added to tyrosine kinase inhibitors (TKIs) to increase the probability of disease control (6). In the past, surgical resection was considered a standard of care for patients with a limited number of BM, while WBRT was used for an extended encephalic involvement with unsatisfying results due to the well-known radio-resistance of renal carcinoma. In recent years, high dose radiotherapy has become the treatment of choice for low number BM to overcome this radioresistance. Stereotactic radiotherapy, a new irradiation technique usually administered in 1 (i.e., radiosurgery) to 5 fractions (i.e., hypofractionated stereotactic radiotherapy), appears to be a proper approach considering its high precision in dose delivering to the tumor, limiting the irradiation of surrounding normal brain tissue $(7,8)$. Randomized trials established radiosurgery over WBRT as the standard of care for patients with 1 to $4 \mathrm{BM}(9,10)$. Of note, some recent prospective and retrospective studies support the possibility to treat also patients with a number of $\mathrm{BM} \geq 5$, obtaining comparable survival benefit in respect to patients with a number of $\mathrm{BM}<5(11,12)$. However, conflicting data about safety and efficacy of the combination of radiotherapy and TKIs for patients with BM from renal carcinoma still remain in the literature $(13,14)$. About the 
issue of $\mathrm{BM}$ in $\mathrm{mRCC}$, some recently published pre-clinical studies suggested the potential activity of new targeted therapies and immune-oncology agents. Of interest, Derosa at al. showed a heterogeneous molecular characterization of metastatic sites versus primary renal carcinoma, with a different expression of programmed-death ligand 1 (PD-L1) and MET on BM, pancreatic metastases, and primary renal lesion (PD-L1 expression: 23\%, 19\%, and 12\%; MET expression: $35 \%, 2 \%$, and $0 \%$ ). These results suggested a biological rationale to use specific systemic therapies, especially in BM that strongly express these targets (15). To date, some case reports revealed a good central nervous system response of VEGF TKI cabozantinib in mRCC patients with $\mathrm{BM}$, probably related to its additional activity against MET (16). However, the efficacy of cabozantinib in this setting still requires exploration.

The introduction of immunotherapy in the therapeutic landscape of mRCC raises important clinical questions also for patients with BM: may immune checkpoint-inhibitors (ICIs) be effective in these patients? May have a sense to combine these new agents with loco-regional therapies to improve both local disease control and survival? Has radiotherapy an additional value or can we skip it? At present, no definitive answers exist to these issues, because large part of the available data derive from retrospective studies.

Flippot et al. provides initial responses in this unexplored field in a recently published BM subgroup analysis of the GETUG-AFU 26 NIVOREN multicenter phase 2 study. The original trial evaluated the safety and efficacy of nivolumab in mRCC patients with a progressive disease after at least one TKI. In the present sub-group analysis, results of patients with asymptomatic BM included in the GETUG-AFU 26 NIVOREN trial and submitted to a dedicated brain evaluation, were reported. Two cohorts were established: patients with untreated BM (cohort A, $\mathrm{n}=39$ patients) and cases with pretreated $\mathrm{BM}$ (cohort $\mathrm{B}$, $\mathrm{n}=34$ patients). The primary objective of this subgroup analysis was the intracranial ORR of nivolumab in cohort A. This primary end point seems to be of particular interest to demonstrate the biological rationale and the potential advantages of using ICIs alone in patients with mRCC and asymptomatic BM, also considering the promising results of some clinical trials in this setting in other solid tumors (17). Growing experience is being collected in non-small cell lung cancer (NSCLC) and melanoma, where BM are more common. At present, only a phase II trial has investigated the efficacy of pembrolizumab in patients with NSCLC and
BM (18). Thirty-nine patients were eligible and a $29.4 \%$ intracranial ORR was observed in the PD-L1-positive cohort with no responses in the negative or not evaluable (0 of 5 patients) PD-L1 status cohort. These results were not confirmed in the subgroup analysis of the Italian nivolumab EAP of squamous and non-squamous NSCLC patients with BM, where an ORR of $17 \%$ and $19 \%$ in the non-squamous and squamous population, was respectively observed $(19,20)$. Preliminary and promising data about the combination of ICIs for the treatment of BM are available for metastatic melanoma. The ORR raises from $15-20 \%$ with ipilimumab, nivolumab or pembrolizumab monotherapy to $40-50 \%$ with the ipilimumab/nivolumab combination as shown in CheckMate 204, a phase 2 singlearm study, and in a further randomized phase 2 trial by Long et al. $(21,22)$.

The results reported by Flippot et al. confirm the limited activity of immune checkpoint inhibitor monotherapy for the treatment of BM in patients with $\mathrm{mRCC}$. The intracranial ORR of nivolumab alone was $12 \%$ (4 of 34 cases) in patients without previous local treatment for BM evaluated in the cohort A, with 13 patients (38\%) having stable intracranial disease as the best response. Of note, the 4 patients with partial response had a unique BM smaller than $10 \mathrm{~mm}$ at baseline. In cohort $\mathrm{B}$, attention should be paid to the fact that most of these patients pretreated for BM, received a further stereotactic radiation therapy $(88 \%)$ due to a local progression. Median intracranial progression-free survival (PFS) was 2.7 months (95\% CI, 2.3 to 4.6 months) in cohort A and 4.8 months (95\% CI, 3.0 to 8.0 months) in cohort $\mathrm{B}$, with prior focal therapy to $\mathrm{BM}$ significantly decreasing the risk of intracranial progression (hazard ratio, 0.49; $95 \%$ CI, 0.26 to 0.92 ). Median overall PFS was similar in the two groups, while 12 -month OS rate was $66.7 \%(95 \%$ CI, $49.6 \%$ to $79.1 \%$ ) and $58.8 \%$ (95\% CI, $40.6 \%$ to $73.2 \%$ ) in cohort $\mathrm{A}$ and cohort $\mathrm{B}$, also due to a more negative baseline prognostic factors selection in cohort B. On the contrary, 19 of 39 patients (49\%) in cohort A and only 11 of 34 patients (32\%) in cohort B had symptoms related to $\mathrm{BM}$, with a corticosteroid use for symptomatic encephalic disease in $51 \%$ and $27 \%$, respectively. The safety profile of nivolumab was acceptable in both cohorts. Overall the results of this trial, also considering the different patient characteristics of cohorts A and B, suggest the possible advantages of combining nivolumab with local treatment, such as radiotherapy, for mRCC with BM. Which radiotherapy technique and when it should be administered is still an open issue. 
The efficacy of concurrent radiosurgery, or hypofractionated stereotactic radiotherapy, and ICIs has been evaluated in patients with BM from solid tumors in recent trials. This combination is theorized to be synergistic for anti-tumor immunity in patients with BM (23). Moreover, due to an eventual "abscopal effect" this combined approach may give a chance of systemic disease control, associated with a decreased incidence of new BM and a favorable survival outcome in selected subgroup of patients (24).

We have to congratulate Flippot and colleagues, because their results support the opportunity to combine different treatment options, in mRCC and BM, to raise the activity of single immune-checkpoint inhibitor. In line with melanoma, the most promising treatment appears the combination of CTLA-4 and PD-L1/PD-1 inhibitors, such as ipilimumab and nivolumab, also considering the possible emerging combination of ICIs with anti-angiogenic agents (25).

\section{Acknowledgments}

None.

\section{Footnote}

Conflicts of Interest: The authors have no conflicts of interest to declare.

Ethical Statement: The authors are accountable for all aspects of the work in ensuring that questions related to the accuracy or integrity of any part of the work are appropriately investigated and resolved.

\section{References}

1. Heng DY, Xie W, Regan MM, et al. Prognostic factors for overall survival in patients with metastatic renal cell carcinoma treated with vascular endothelial growth factortargeted agents: results from a large, multicenter study. J Clin Oncol 2009;27:5794-9.

2. Culine S, Bekradda M, Kramar A, et al. Prognostic factors for survival in patients with brain metastases from renal cell carcinoma. Cancer 1998;83:2548-53.

3. Gore ME, Szczylik C, Porta C, et al. Final results from the large sunitinib global expanded-access trial in metastatic renal cell carcinoma. Br J Cancer 2015;113:12-9.

4. Gore ME, Hariharan S, Porta C, et al. Sunitinib in metastatic renal cell carcinoma patients with brain metastases. Cancer 2011;117:501-9.

5. Chevreau C, Revaud A, Escudier B, et al. A phase II trial of sunitinib in patients with renal cell cancer and untreated brain metastases. Clin Genitourin Cancer 2014;12:50-4.

6. De Meerleer G, Khoo V, Escudier B, et al. Radiotherapy for renal-cell carcinoma. Lancet Oncol 2014;15:e170-e177.

7. Ippen FM, Mahadevan A, Wong ET, et al. Stereotactic radiosurgery for renal cancer brain metastasis: prognostic factors and the role of whole-brain radiation and surgical resection. J Oncol 2015;2015:636918.

8. Lesueur P, Lequense J, Barraux V, et al. Radiosurgery of hypofractionated stereotactic radiotherapy for brain metastases from radioresistent primaries (melanoma and renal cancer). Radiat Oncol 2018;13:138.

9. Aoyama H, Shirato H, Tago M, et al. Stereotactic radiosurgery plus whole-brain radiation therapy vs stereotactic radiosurgery alone for treatment of brain metastases: a randomized controlled trial. JAMA 2006;295:2483-91.

10. Brown PD, Jeackle K, Ballman KV, et al. Effect of radiosurgery alone vs radiosurgery with whole brain radiation therapy on cognitive function in patient with 1 to 3 brain metastases. A randomized clinical trial. JAMA 2016;316:401-9.

11. Yamamoto M, Serizawa T, Shuto T, et al. Stereotactic radiosurgery for patients with multiple brain metastases (JLGK0901): A multi-institutional prospective oservational study. Lancet Oncol 2014;15:387-95.

12. Hughes RT, Masters AH, McTyre ER, et al. Initial SRS for Patients With 5 to 15 Brain Metastases: Results of a Multi-Institutional Experience. Int J Radiat Oncol Biol Phys 2019;104:1091-8.

13. Staehler M, Haseke N, Stadler T, et al. Feasibility and effects of high-dose hypofractionated radiation therapy and simultaneous multi-kinase inhibition with sunitinib in progressive metastatic renal cell cancer. Urol Oncol 2012;30:290-3.

14. Juloori A, Miller JA, Parsai S, et al. Overall survival and response to radiation and targeted therapies among patients with renal cell carcinoma brain metastases. J Neurosurg 2019:1-9. [Epub ahead of print].

15. Derosa L, Le Teuff G, Khordahi M, et al. Inter and intratumor heterogeneity of PD-L1 and MET expression in metastatic renal cell carcinoma (mRCC). J Clin Oncol 2017;35:abstr 4569.

16. Ciccarese C, Iacovelli R, Mosillo C, et al. Exceptional Response to Cabozantinib of Rapidly Evolving Brain Metastases of Renal Cell Carcinoma: A Case Report 


\section{Page 4 of 4}

and Review of the Literature. Clin Genitourin Cancer 2018;16:e1069-71.

17. El Rassy E, Botticella A, Kattan J, et al. Non-small cell lung cancer brain metastases and the immune system: From brain metastases development to treatment. Cancer Treat Rev 2018;68:69-79.

18. Goldberg SB, Gettinger SN, Mahajan A, et al. Durability of brain metastasis response and overall survival in patients with non-small cell lung cancer (NSCLC) treated with pembrolizumab. J Clin Oncol 2018;36:abstr 2009.

19. Cortinovis D, Chiari R, Catino A, et al. Italian Cohort of the Nivolumab EAP in Squamous NSCLC: Efficacy and Safety in Patients With CNS Metastases. Anticancer Res 2019;39:4265-71.

20. Crinò L, Bronte G, Bidoli P, et al. Nivolumab and brain metastases in patients with advanced non-squamous nonsmall cell lung cancer. Lung Cancer 2019;129:35-40.

21. Tawbi HA, Forsyth PA, Algazi A, et al. Combined Nivolumab and Ipilimumab in Melanoma Metastatic to

Cite this article as: Bracarda S, Mosillo C, Trippa F, Urbano F, Maranzano E, Caserta C. No paradigm changes with checkpoint inhibitor monotherapy in patients with metastatic renal cell carcinoma and brain metastases. Ann Transl Med 2019;7(22):612. doi: 10.21037/atm.2019.11.50

\section{Bracarda et al. Activity of nivolumab for renal cell carcinoma BM}

the Brain. N Engl J Med 2018;379:722-30.

22. Long GV, Atkinson V, Lo S, et al. Combination nivolumab and ipilimumab or nivolumab alone in melanoma brain metastases: a multicentre randomised phase 2 study. Lancet Oncol 2018;19:672-81.

23. Trapani S, Manicone M, Sikokis A, et al. Effectiveness and safety of "real" concurrent stereotactic radiotherapy and immunotherapy in metastatic solid tumors: a systematic review. Crit Rev Oncol Hematol 2019;142:9-15.

24. Chen L, Douglass J, Kleinberg L, et al. Concurrent Immune Checkpoint Inhibitors and Stereotactic Radiosurgery for Brain Metastases in Non-Small Cell Lung Cancer, Melanoma, and Renal Cell Carcinoma. Int J Radiat Oncol Biol Phys 2018;100:916-25.

25. Bracarda S, Porta C, Sabbatini R, et al. Angiogenic and immunological pathways in metastatic renal cell carcinoma: A counteracting paradigm or two faces of the same medal? The GIANUS Review. Crit Rev Oncol Hematol 2019;139:149-57. 